Check for updates

Cite this: Mater. Chem. Front., 2019, 3, 2529

\section{Correction: A series of red iridium(III) complexes using flexible dithiocarbamate derivatives as ancillary ligands for highly efficient phosphorescent OLEDs}

\author{
Guang-Zhao Lu, ${ }^{\text {ab }}$ Ruixia Wu, ${ }^{c}$ Liang Liu, ${ }^{a}$ Liang Zhou, *c You-Xuan Zheng, *a \\ Wen-Wei Zhang, ${ }^{* a}$ Jing-Lin Zuo and Hongjie Zhang ${ }^{c}$
}

DOI: $10.1039 / c 9 q m 90045 d$

Correction for 'A series of red iridium(III) complexes using flexible dithiocarbamate derivatives as ancillary ligands for highly efficient phosphorescent OLEDs' by Guang-Zhao Lu et al., Mater. Chem. Front., 2019,

rsc.li/frontiers-materials

This correction is being issued to draw the readers' attention to the authors' closely related papers, published at a similar time in Chemical Science ${ }^{1}$ and Journal of Materials Chemistry $C,{ }^{2,3}$ which should have been cited in this Materials Chemistry Frontiers paper. The authors understand that they should have notified the journal's editors about the related manuscripts when this Materials Chemistry Frontiers paper was under review.

All four papers report cyclometalated iridium(III) complexes that contain a four membered ring based on the same Ir-S-C-S backbone. However, the complexes reported in each paper have different cyclometalated ligands and/or different dithiocarbamate derivatives as the main ligands and ancillary ligands, respectively.

In this Materials Chemistry Frontiers paper, the authors reported five complexes using 1-(4-(trifluoromethyl)phenyl)isoquinoline as the main ligand and five dithiocarbamate derivatives as ancillary ligands. In ref. 1 and 2, five different complexes were reported where different ancillary ligands were used but with 4-(4-(trifluoromethyl)phenyl)quinazoline as the main ligand. In ref. 3, three complexes were reported where different main ligands were used but with the same dithiocarbamate derivative as the ancillary ligand.

Therefore, although all the papers reported iridium(III) complexes with similar structures, these materials show different photophysical properties and device performances. However, ref. 1-3 should have been cited in this Materials Chemistry Frontiers paper.

The authors also regret that there are portions of unattributed text overlap in the Introduction and Results and discussion sections with other papers published by the authors, including ref. 1 and 2.

The Royal Society of Chemistry apologises for these errors and any consequent inconvenience to authors and readers.

\title{
References
}

1 G.-Z. Lu, N. Su, H.-Q. Yang, Q. Zhu, W.-W. Zhang, Y.-X. Zheng, L. Zhou, J.-L. Zuo, Z.-X. Chen and H.-J. Zhang, Chem. Sci., 2019, 10, 3535-3542.

2 G.-Z. Lu, X. Li, L. Liu, L. Zhou, Y.-X. Zheng, W.-W. Zhang, J.-L. Zuo and H. Zhang, J. Mater. Chem. C, 2019, 7, 3862-3868.

3 G.-Z. Lu, Z.-L. Tu, L. Liu, W.-W. Zhang and Y.-X. Zheng, J. Mater. Chem. C, 2019, 7, 7273-7278.

\footnotetext{
${ }^{a}$ State Key Laboratory of Coordination Chemistry, Collaborative Innovation Center of Advanced Microstructures, Jiangsu Key Laboratory of Advanced Organic Materials, School of Chemistry and Chemical Engineering, Nanjing University, Nanjing 210093, P. R. China. E-mail: yxzheng@nju.edu.cn

${ }^{b}$ Shenzhen Key Laboratory of Polymer Science and Technology, College of Materials Science and Engineering, Shenzhen University, Shenzhen 518060, P. R. China. E-mail: gzhlu@szu.edu.cn

${ }^{c}$ State Key Laboratory of Rare Earth Resource Utilization, Changchun Institute of Applied Chemistry, Chinese Academy of Sciences, Changchun 130022, P. R. China. E-mail:zhoul@ciac.ac.cn
} 